\title{
MODELAGEM CINÉTICA DA PRODUÇÃO DE PROPENO A PARTIR DE ACETONA EM UMA ÚNICA ETAPA
}

\author{
H. ENZWEILER ${ }^{1}$, E. B. COUTINHO ${ }^{2}$ e M. SCHWAAB ${ }^{3}$ \\ ${ }^{1}$ Universidade Estadual de Maringá, Departamento de Engenharia Química \\ ${ }^{2}$ Universidade Federal do Rio Grande do Sul, Departamento de Físico-Química \\ ${ }^{3}$ Universidade Federal Santa Maria, Departamento de Engenharia Química \\ E-mail para contato: marcioschwaab@gmail.com
}

\begin{abstract}
RESUMO - O propeno pode ser sintetizado diretamente a partir da acetona, que pode ser obtida a partir de fontes renováveis como a biomassa. O processo em uma única etapa une em um reator a hidrogenação de acetona, seguida da desidratação do isopropanol formado. Para tanto é empregada como catalisador mistura de óxido de $\mathrm{Cu} / \mathrm{Zn} / \mathrm{Al}$ derivado de material do tipo hidrotalcita e de zeólita Beta. Para os testes reacionais foi utilizado planejamento de experimentos, variando as condições de alimentação e temperatura de reação. O processo completo de conversão de acetona em propeno foi realizado em onze condições distintas; e a fração de olefina foi de até $65 \%$ dentre os compostos orgânicos. Um modelo cinético, baseado em mecanismos do tipo LangmuirHinshelwood, eficaz para a representação das frações molares dos compostos orgânicos foi desenvolvido. Simulações do reator tubular usando este modelo cinético foram empregadas no estudo do efeito das variáveis de processo nos produtos da reação.
\end{abstract}

\section{INTRODUÇÃO}

O propeno é um composto químico de alto valor agregado e largamente empregado como matéria-prima na indústria. Atualmente a maior parte do propeno utilizado provém do petróleo, porém a busca por fontes renováveis para esta olefina vem recebendo cada vez mais atenção. Por sua vez, a acetona é um composto orgânico oxigenado de três carbonos amplamente utilizada como solvente. Industrialmente a acetona é obtida principalmente como subproduto do processo de síntese do fenol. Entretanto, a acetona também pode ser obtida a partir de fontes renováveis, como subproduto da fermentação para produção de butanol ou ainda na fase aquosa do bio-óleo, obtido por pirólise de biomassa.

A acetona pode ser convertida através de uma rota simples em propeno (Ohkubo et al., 2011), produto com maior valor de mercado. O estudo do mecanismo reacional da síntese de propeno a partir da acetona é o principal objetivo deste trabalho. Para tanto, a rota química proposta é a reação de hidrogenação de acetona, em presença de um óxido misto de $\mathrm{Cu} / \mathrm{Zn} / \mathrm{Al}$, seguida da desidratação do isopropanol formado, com zeólita Beta, através de um processo em uma única etapa. 


\section{MATERIAIS E MÉTODOS}

\subsection{Síntese e caracterização dos catalisadores}

Como catalisador para a reação de hidrogenação (HT) utilizou-se o óxido misto de $\mathrm{Cu} / \mathrm{Zn} / \mathrm{Al}$ derivado de material do tipo hidrotalcita. O material precursor do tipo hidrotalcita de $\mathrm{Cu} / \mathrm{Zn} / \mathrm{Al}$ foi sintetizado através do método da coprecipitação a pH variável (Souza et al., 2008). As proporções entre os reagentes foram escolhidas de forma que a razão de $(\mathrm{Cu}+\mathrm{Zn}) / \mathrm{Al}$ fosse igual a 3 e a razão molar de $\mathrm{Cu} / \mathrm{Zn}$ fosse igual a 0,2 . Para a obtenção do óxido misto, o material precursor foi calcinado em mufla a $450{ }^{\circ} \mathrm{C}, 5{ }^{\circ} \mathrm{C} / \mathrm{min}$, por 10 horas. Para a reação de desidratação, optou-se pelo emprego de zeólita Beta na forma ácida $(\mathrm{H}-\beta)$ como catalisador. A zeólita Beta foi sintetizada como descrito em Enzweiler et al. (2013), utilizando sílica extraída de cinzas de casca de arroz, pseudoboemita e hidróxido de tetraetilamônio como fontes de silício e alumínio e direcionador de estrutura.

A composição química do material precursor do óxido misto foi determinada por espectrofotometria de absorção atômica por chama, em equipamento 200 Series AA (Agilent Technologies), com chama de acetileno/ar sintético, para cobre e zinco e acetileno/ $\mathrm{N}_{2} \mathrm{O}$, para alumínio. A estrutura cristalina dos catalisadores foi identificada por difração de raios-X, em um difratômetro de raios-X Rigaku modelo Miniflex, com radiação $\mathrm{Cu} \mathrm{K \alpha}$ e fonte de energia com $30 \mathrm{kV}$ e $10 \mathrm{~mA}$. A adsorção/dessorção estática de $\mathrm{N}_{2}$ a $77 \mathrm{~K}$ foi utilizada para análise das propriedades texturais dos catalisadores, em equipamento ASAP 2020 (Micromeritics).

\subsection{Testes catalíticos}

Os testes catalíticos eram realizados em unidade de reação em leito fixo com fluxo contínuo. A unidade era constituída de forno, com controle digital de temperatura e reator de vidro em "U". Os reagentes e produtos da reação eram analisados por cromatografia gasosa, Shimadzu (GC-17A) equipado com coluna capilar HP-PLOT-Q (Agilent Technologies) e com detector tipo ionização em chama (FID). A acetona (Neon, pureza $\geq 99,5 \%$ ) era alimentada através do borbulhamento de $\mathrm{N}_{2}$ em um saturador, contendo o reagente, mantido a temperatura controlada em banho termostático.

Nos experimentos de produção de propeno a partir de acetona em uma única etapa $40 \mathrm{mg}$ de uma mistura mecânica dos catalisadores, em três diferentes proporções, eram acondicionados no reator. $\mathrm{O}$ óxido misto era reduzido in situ sob fluxo de $\mathrm{H}_{2}$ e inerte a $400{ }^{\circ} \mathrm{C}$. As condições reacionais eram variadas de acordo com um planejamento experimental mínimo fatorial a dois níveis com triplicata no ponto central. Os experimentos eram realizados em seis temperaturas de reação $\left(130,150,160,170,180\right.$ e $\left.200{ }^{\circ} \mathrm{C}\right)$. Em todas as condições experimentais a vazão de $\mathrm{N}_{2}$ passando pelo saturador era mantida em $12 \mathrm{~mL} / \mathrm{min}$. As demais variáveis operacionais eram fixadas de acordo com o planejamento experimental apresentado na Tabela 1. 
Tabela 1 - Planejamento experimental para produção de propeno a partir de acetona

\begin{tabular}{ccccc}
\hline Experimento & $\begin{array}{c}\text { Temperatura do } \\
\text { saturador }\left({ }^{\circ} \mathrm{C}\right)\end{array}$ & $m_{H T} / m_{H-\beta}$ & $\begin{array}{c}\text { Vazão de } \mathrm{H}_{2} \\
(\mathrm{~mL} / \mathrm{min})\end{array}$ & $\begin{array}{c}\text { Vazão de } \mathrm{H}_{2} / \mathrm{N}_{2} \\
(\mathrm{~mL} / \mathrm{min})\end{array}$ \\
\hline E1 & $10(-1)$ & $4(-1)$ & $40(1)$ & $23(1)$ \\
E2 & $10(-1)$ & $6(1)$ & $23(-1)$ & $23(1)$ \\
E3 & $20(1)$ & $6(1)$ & $40(1)$ & $23(1)$ \\
E4 & $10(-1)$ & $4(-1)$ & $23(-1)$ & $0(-1)$ \\
E5 & $10(-1)$ & $6(1)$ & $40(1)$ & $0(-1)$ \\
E6 & $20(1)$ & $4(-1)$ & $23(-1)$ & $23(1)$ \\
E7 & $20(1)$ & $4(-1)$ & $40(1)$ & $0(-1)$ \\
E8 & $20(1)$ & $6(1)$ & $23(-1)$ & $0(-1)$ \\
E9 & $15(0)$ & $5(0)$ & $31,5(0)$ & $11,5(0)$ \\
E10 & $15(0)$ & $5(0)$ & $31,5(0)$ & $11,5(0)$ \\
E11 & $15(0)$ & $5(0)$ & $31,5(0)$ & $11,5(0)$ \\
\hline
\end{tabular}

(Valor normalizado das variáveis)

\subsection{Modelos cinéticos}

As velocidades das reações globais $\left(r_{i}\right)$ foram obtidas para as três reações envolvidas: hidrogenação de acetona (R1), desidratação de isopropanol formando propeno (R2) e hidrogenação de propeno a propano (R3). Sendo que as reações R1 e R3 ocorrem na superfície do catalisador metálico (HT), e a reação R2 sobre o catalisador ácido (H- $\beta$ ). Inicialmente considerou-se que o sistema reacional, apesar da presença dos catalisadores heterogêneos, seguia o comportamento de reações homogêneas. O modelo resultante dessa hipótese é chamado de pseudo-homogêneo (HD1). Contudo estas velocidades de reação também podem ser obtidas a partir dos mecanismos envolvidos nas reações heterogêneas (HD2), através do método de Langmuir-Hinshelwood-Hougen-Watson (Figueiredo e Ribeiro, 1989), em que se consideram as três reações (R1, R2 e R3), com todas as suas etapas intermediárias de adsorção, dessorção e reação de superfície, totalizando 10 etapas intermediárias distintas, Figura 1. A mistura de catalisadores com diferentes funcionalidades possibilitou a presença de dois tipos de sítios ativos distintos no meio reacional; metálicos $\left(*_{\mathrm{M}}\right)$ do óxido misto e ácidos $\left({ }^{*} \mathrm{~A}\right)$ da zeólita Beta.

$$
\begin{gathered}
\text { Acetona }+ \text { Hidrogênio } \rightleftharpoons \text { Isopropanol } \\
\text { Isopropanol } \rightleftharpoons \text { Propeno }+ \text { Água } \\
\text { Propeno }+ \text { Hidrogênio } \rightleftharpoons \text { Propano }
\end{gathered}
$$

Devido à complexidade do mecanismo, apenas um modelo cinético (HD2) foi utilizado no ajuste aos dados experimentais, além do modelo de reação pseudo-homogênea (HD1). As taxas $\left(r_{1}\right),\left(r_{2}\right)$ e $\left(r_{3}\right)$ para o cinética heterogênea (HD2) foram formuladas considerando como etapas determinantes de velocidade as reações intermediárias (R1.3), (R2.2) e (R3.2), que são as reações de superfície. 


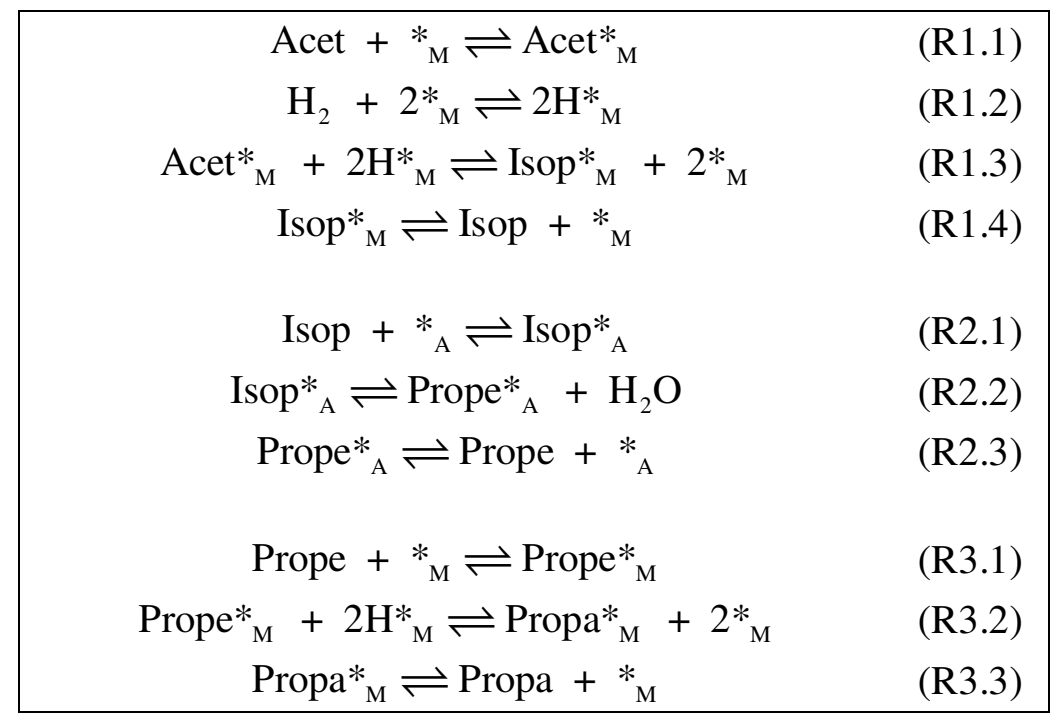

Figura 1 - Mecanismo cinético proposto para produção de propeno a partir de acetona.

\subsection{Estimação de parâmetros}

O sistema reacional foi representado por modelos típicos de reator tubular com fluxo empistonado. O modelo do reator composto por um sistema de equações diferenciais foi resolvido numericamente com o auxílio da rotina DASSL (Petzold, 1989). Os modelos propostos foram ajustados aos dados experimentais das frações molares dos compostos na fração orgânica na saída do reator, os parâmetros estimados foram as constantes cinéticas $\left(\ln \left(\mathrm{k}_{\mathrm{m}}{ }^{\text {Tref }}\right)\right.$ e $\mathrm{E}_{\mathrm{m}} /\left(\mathrm{R} . \mathrm{T}_{\mathrm{ref}}\right)$ ) na forma da equação de Arrhenius reparametrizada, Equação 1 (Schwaab e Pinto, 2007), e as constantes de equilíbrio das etapas intermediárias $\left(\ln \left(K_{n}^{\text {Tref }}\right)\right.$ e $\left.\Delta H_{n} /\left(R \cdot T_{\text {ref }}\right)\right)$. As constantes de equilíbrio são funções da temperatura, assim como as constantes cinéticas, e variam conforme a equação de van't Hoff reparametrizada, Equação 2. A temperatura de referência foi de $160{ }^{\circ} \mathrm{C}$. Na estimação de parâmetros os valores das constantes de equilíbrio das reações globais (R1, R2, R3) foram calculados a partir de dados termodinâmicos. A estimação foi realizada através da minimização da função objetivo de mínimos quadrados ponderados, com o uso de um algoritmo híbrido de otimização composto por um método estocástico acoplado a um método determinístico, em que a melhor solução encontrada pelo método do Enxame de Partículas (Kennedy e Eberhart, 1995) era utilizada como estimativa inicial para um método do tipo Gauss-Newton.

$$
\begin{aligned}
& k_{i, m}=\exp \left[\ln \left(k_{i, m}^{T_{r e f}}\right)+\frac{E_{i, m}}{R T_{r e f}}\left(\frac{T-T_{r e f}}{T}\right)\right] \\
& K_{i, n}=\exp \left[\ln \left(K_{i, n}^{T_{r, f}}\right)+\frac{\Delta H_{i, n}}{R T_{r e f}}\left(\frac{T-T_{r e f}}{T}\right)\right]
\end{aligned}
$$

O efeito das variáveis operacionais foi analisado através do modelo cinético. Para tanto, 


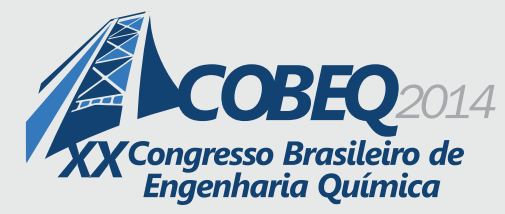

foram escolhidas as variáveis operacionais a razão molar entre hidrogênio e acetona na alimentação, a fração molar de acetona na alimentação e a velocidade mássica espacial por hora. A vazão molar de acetona na alimentação foi mantida fixa e as outras variáveis foram calculadas a partir das variáveis operacionais.

\section{RESULTADOS E DISCUSSÃO}

\subsection{Produção de propeno a partir de acetona}

$\mathrm{O}$ óxido misto de $\mathrm{Cu} / \mathrm{Zn} / \mathrm{Al}$ derivado de material do tipo hidrotalcita apresentou razão molar $\mathrm{Cu} / \mathrm{Zn}$ de 0,19 e $(\mathrm{Cu}+\mathrm{Zn}) / \mathrm{Al}$ de 1,9 , área específica de $110 \mathrm{~m}^{2} / \mathrm{g}$, volume total de poros de $0,435 \mathrm{~cm}^{3} / \mathrm{g}$ e raio médio de poro de $82 \AA$. A caracterização de sua estrutura cristalina indicou indicando a presença de material tipo hidrotalcita após a síntese e do óxido misto com a presença das fases $\mathrm{CuO}$ e $\mathrm{ZnO}$, além de $\mathrm{ZnAlO}_{4}$ após calcinação. A caracterização do catalisador ácido por difração de raios- $X$ indicou a formação de estrutura cristalina da zeólita Beta. A forma ácida da zeólita Beta apresentou área específica de $395 \mathrm{~m}^{2} / \mathrm{g}$, volume total de poros de $0,246 \mathrm{~cm}^{3} / \mathrm{g} \mathrm{e}$ raio médio de poros de $36 \AA$.

Na produção de propeno a partir de acetona ocorre primeiramente a hidrogenação, seguida da desidratação do álcool formado, realizada em um leito catalítico contendo mistura mecânica dos catalisadores. Os experimentos foram efetuados de maneira independente e as variáveis operacionais foram ajustadas de acordo com o planejamento experimental. A maior conversão de acetona na temperatura de $150{ }^{\circ} \mathrm{C}$ foi observada no experimento E1, seguido do experimento E7. Em ambos os casos a concentração de hidrogênio no meio reacional é a mais elevada, indicando que a conversão é fortemente influenciada por esta variável operacional. Com o aumento da temperatura até $200{ }^{\circ} \mathrm{C}$, na maioria das condições experimentais, atingiu-se a conversão completa da acetona.

Em todos os experimentos o éter di-isopropílico foi encontrado apenas em pequenas quantidades, inferior a $1,8 \%$, e em temperaturas até $170{ }^{\circ} \mathrm{C}$. Portanto, a reação de formação do éter do modelo cinético do processo de produção de propeno a partir de acetona foi desconsiderada, visto que este composto é formado em pequenas quantidades e não é um produto de interesse deste estudo. A formação de isopropanol também é observada em todos os experimentos, sendo este consumido totalmente nas temperaturas mais elevadas. A presença do isopropanol confirma que a produção de propeno ocorreu de acordo com a rota química esperada. A formação de propano é cineticamente favorecida com o aumento da temperatura. Em muitos casos ocorre formação preferencial do alcano que da olefina, principalmente a $200{ }^{\circ} \mathrm{C}$, temperatura onde se obtiveram as maiores conversões. Por outro lado, nos experimentos E4, E5, E6, E8 e E10, o produto preferencial é o propeno em todas as temperaturas de reação. Os experimentos E5, E6 e E8 correspondem às condições com maior rendimento a propeno, acima de $50 \%$, a $200{ }^{\circ} \mathrm{C}$. No experimento E5 atingiu-se $65 \%$ de propeno na fração orgânica.

Os resultados indicam que há uma série de fatores envolvidos no processo de produção de propeno a partir da acetona que, muitas vezes, podem favorecer a formação a de propeno. $\mathrm{O}$ 


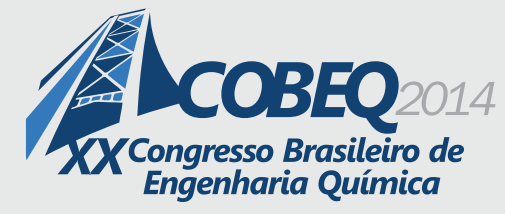

aumento da fração de hidrogênio, de maneira geral, aumenta a conversão global de acetona, porém também contribui para a hidrogenação do propeno formado. Não foi possível observar a influência da razão mássica entre os catalisadores, talvez em razão da variação ter sido pequena ou por este efeito ter sido ocultado por efeitos de outras variáveis operacionais

\subsection{Modelagem cinética}

Para a estimação dos parâmetros cinéticos das reações de hidrogenação e de desidratação em uma única etapa foram empregados os dados experimentais obtidos nas condições reacionais de E1 a E11, em todas as temperaturas. Verificou-se que o modelo que leva em conta as etapas de adsorção e dessorção (HD2) proporcionou menor valor da função objetivo que o modelo de reação pseudo-homogênea (HD1), indicando que o modelo mais completo é o mais adequado para representar os dados experimentais.

Foram testados os diferentes modelos cinéticos, variando-se também a quantidade de parâmetros em cada modelo, sendo a influência destes parâmetros observada no valor da função objetivo e na confiabilidade dos demais parâmetros estimados, partindo-se do modelo mais simples, o pseudo-homogêneo. Dessa forma, obteve-se o modelo simplificado (HD2S), Tabela 2, com menor número de parâmetros, que pode representar adequadamente o processo sem prejuízos à qualidade do ajuste. Nesse sentido foi possível a redução de 20 para 12 parâmetros a serem estimados. Os valores dos parâmetros e seus respectivos desvios padrão são apresentados na Tabela 3. Embora a estimação dos parâmetros e obtenção do modelo para o processo completo de produção de propeno a partir de acetona em uma única etapa sejam tarefas bastante complexas, o ajuste obtido pelo modelo proposto é aceitável para representar as frações molares de acetona, de isopropanol e de propano e muito bom para a fração molar de propeno, produto de interesse.

Tabela 2 - Modelos cinéticos HD2 e HD2S

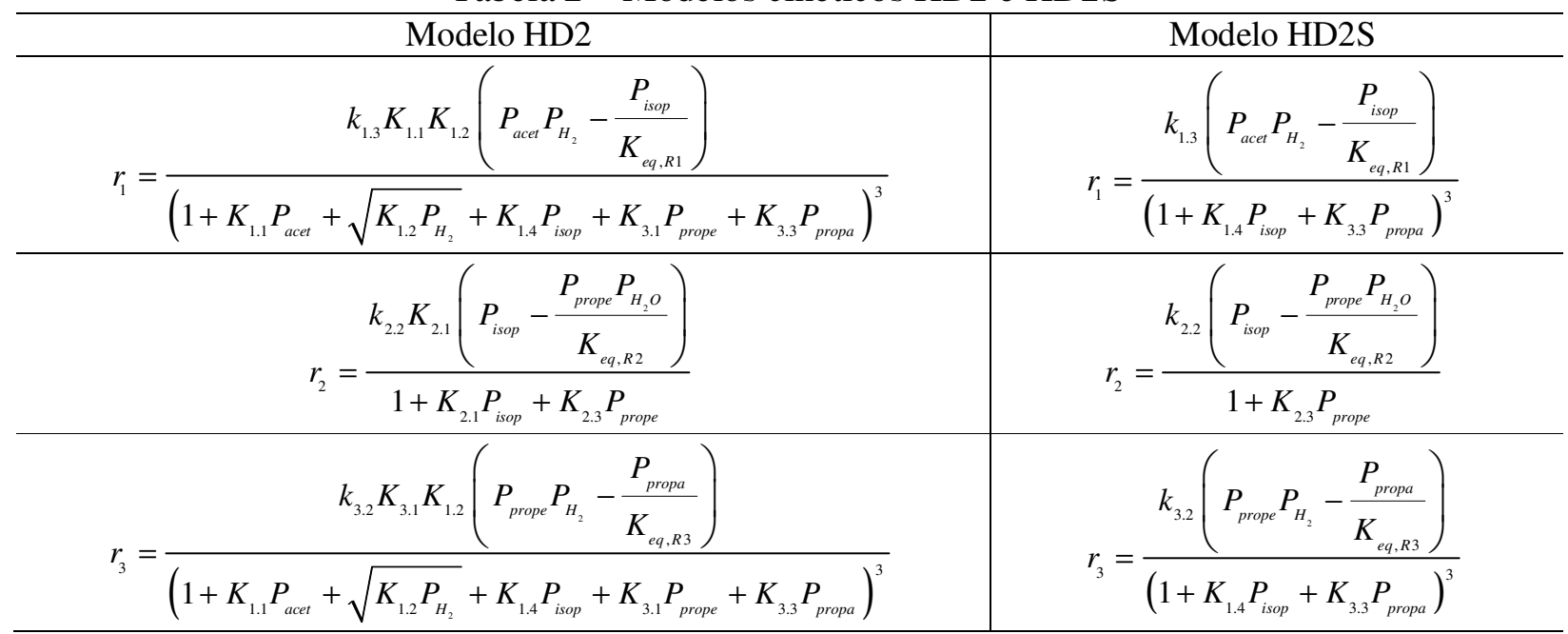


Tabela 3 - Parâmetros cinéticos e desvio padrão para o modelo HD2S

\begin{tabular}{|c|c|c|c|c|c|}
\hline Parâmetro & Valor & Desvio Padrão & Parâmetro & Valor & Desvio Padrão \\
\hline $\ln \left(k_{1.3}^{T_{r f f}}\right)$ & 4,818 & 0,622 & $E_{1.3} / R T_{r e f}$ & 128,343 & 0,001 \\
\hline $\ln \left(K_{1.4}^{T_{r f f}}\right)$ & 4,368 & 1,389 & $\Delta H_{1.4} / R T_{r e f}$ & 5,074 & 10,798 \\
\hline $\ln \left(k_{2.2}^{T_{, j}}\right)$ & 14,746 & 24,963 & $E_{2.2} / R T_{r e f}$ & 34,313 & 22,699 \\
\hline $\ln \left(K_{2.3}^{T_{f f}}\right)$ & 18,365 & 24,908 & $\Delta H_{2.3} / R T_{r f}$ & $-15,911$ & 23,961 \\
\hline $\ln \left(k_{3.2}^{T_{, j}}\right)$ & 6,109 & 0,621 & $E_{3.2} / R T_{r f f}$ & 88,267 & 9,041 \\
\hline $\ln \left(K_{3.3}^{T_{r f}}\right)$ & 7,058 & 0,015 & $\Delta H_{3.3} / R T_{r e f}$ & 16,807 & 3,540 \\
\hline
\end{tabular}

A partir do modelo HD2S, mais adequado, e dos parâmetros estimados foi possível analisar melhor a influência das variáveis do processo sobre frações molares de propeno e propano formados. Há o deslocamento do equilíbrio da reação de hidrogenação, causado pelo consumo do isopropanol na reação simultânea de desidratação. A redução da fração molar de acetona na alimentação provoca o aumento da fração de propeno, em temperaturas inferiores a $185^{\circ} \mathrm{C}$, porém em temperaturas superiores ocorre o favorecimento da formação de propano. A menor velocidade mássica espacial favorece a formação dos produtos, contudo, em temperaturas superiores a $190^{\circ} \mathrm{C}$, o maior tempo de residência provoca a hidrogenação do propeno em maior extensão.

\section{CONCLUSÃO}

Experimentalmente, o processo de produção de propeno a partir de acetona em uma única etapa foi avaliado em diferentes condições experimentais. A fração molar de propeno obtido constituiu até $65 \%$ da fração orgânica do meio reacional, indicando que este processo é viável para a produção da olefina. Um modelo de cinética heterogênea simplificado, que possibilita boa predição das frações molares dos compostos na fração orgânica na saída do reator foi obtido. A grande vantagem deste modelo é a redução da complexidade das equações e do número de parâmetros a serem estimados, aumentando a confiabilidade dos valores obtidos na estimação, sem limitar a qualidade da predição alcançada.

\section{AGRADECIMENTOS}

Os autores agradecem à Fundação de Amparo à Pesquisa do Estado do Rio Grande do Sul (FAPERGS) e a Secretaria de Ciência Tecnologia e Inovação do RS (SCIT-RS).

\section{NOMENCLATURA}
composto*
Espécie adsorvida
$E_{i . m} / R T_{\text {ref }}$
Parâmetro cinético, razão entre a energia de ativação da reação $\left(E_{i . m}\right)$ e o produto entre a constante dos gases $(R)$ e a temperatura de referência $\left(T_{r e f}\right)$ 
$\Delta H_{i . n} / R T_{\text {ref }} \quad$ Parâmetro de equilíbrio, razão entre a variação da entalpia da reação $\left(\Delta H_{i . n}\right) \mathrm{e}$ o produto entre a constante dos gases $(R)$ e a temperatura de referência $\left(T_{\text {ref }}\right)$

$K_{e q, R_{i}} \quad$ Constante de equilíbrio da reação global $R_{i}$

$k_{i . m}$

Constante cinética da reação i.m, sendo $i$ a reação global e $m$ a etapa considerada limitante

$K_{i, n} \quad$ Constante de equilíbrio da etapa i.n, sendo $n$ a etapa intermediária em equilíbrio

$\ln \left(k_{i . m}^{T_{r e f}}\right) \quad$ Parâmetro cinético, logaritmo natural da velocidade específica da reação i.m, sendo $i$ a reação global e $m$ a etapa considerada limitante, na temperatura de referência

$\ln \left(K_{i . n}^{T_{r e f}}\right) \quad$ Parâmetro de equilíbrio, logaritmo natural da constante de equilíbrio da etapa i.n, sendo $n$ a etapa intermediária em equilíbrio, na temperatura de referência

$m_{H T} / m_{H-\beta}$

$P_{j} \quad$ Pressão parcial do composto $j$

$r_{i} \quad$ Velocidade da reação $i$

\section{REFERÊNCIAS}

ENZWEILER, H.; COUTINHO, E. B.; SCHWAAB, M. Cinzas de casca de arroz como fonte alternativa de silício para a síntese de zeólita beta. REGET, v. 17, p. 3284-3292, 2013.

FIGUEIREDO, J. L.; RIBEIRO, F. R. Catálise heterogênea. Fundação Calouste Gulbenkian, Lisboa, 1989.

KENNEDY, J.; EBERHART, R. Particle swarm optimization. In: Proceedings of the IEEE International Conference on Neural Networks, Perth, Australia, v. 4, p. 1942-1948, 1995.

OHKUBO, T.; FUJIWARA, K. FUJITA, T. Mitsui Chemicals Inc (JP). Olefin production process. US 2011/0230696 A1, 22 set. 2011.

PETZOLD, L. R. DASSL Code (Differential Algebraic System Solver), Computing and Mathematics Research Division, Lawrence Livermore National Laboratory, Livermore, CA, USA, 1989.

SCHWAAB, M.; PINTO, J. C. Optimum reference temperature for reparameterization of the Arrhenius equation. Part 1: Problems involving one kinetic constant. Chem Eng Sci, v. 62, p. 2750-2764, 2007.

SOUZA, M. M. V. M.; FERREIRA, K. A.; MACEDO NETO, O. R.; RIBEIRO, N. F. P.; SCHMAL, M. Copper-based catalysts prepared from hydrotalcite precursors for shift reaction at low temperatures. Catal Today, v. 133-135, p. 750-754, 2008. 